\title{
Splenectomy Outcomes in Relapsed or Refractory Immune Thrombocytopenia according to First- Line Intravenous Immunoglobulin Response
}

\author{
Daehun Kwag Jae-Ho Yoon Gi June Min Sung-Soo Park Silvia Park \\ Sung-Eun Lee Byung-Sin Cho Ki-Seong Eom Yoo-Jin Kim Hee-Je Kim \\ Seok Lee Chang-Ki Min Seok-Goo Cho Dong-Wook Kim Jong Wook Lee \\ Department of Hematology, Catholic Hematology Hospital and Leukemia Research, Seoul St. Mary's Hospital, \\ College of Medicine, The Catholic University of Korea, Seoul, Republic of Korea
}

\section{Keywords}

Immune thrombocytopenia · Refractory - Splenectomy ·

Intravenous immunoglobulin · Salvage treatment

\begin{abstract}
Objectives: Although splenectomy has long been secondline option for immune thrombocytopenia (ITP) patients, an indicator that reliably predicts the efficacy of splenectomy is still being explored. We investigated the treatment outcomes of splenectomy as a second-line therapy for relapsed/ refractory ITP according to first-line intravenous immunoglobulin (IVIG) responses. Methods: Fifty-two adult patients treated with splenectomy as second-line therapy for ITP between 2009 and 2019 were included, and they were classified according to first-line IVIG responses (no response to IVIG: nonresponders; only transient IVIG response shorter than 4 weeks: poor responders; IVIG response for a longer period; stable responders). The efficacy of splenectomy was analyzed in the three subgroups. Results: Of the 52 patients, 10 were IVIG nonresponders, 34 were poor responders, and the remaining 8 were stable responders. Response to splenectomy was observed in $50.0 \%$ of IVIG nonresponders, $94.1 \%$ of poor responders, and $100 \%$ of stable responders ( $p$
\end{abstract}

Karger@karger.com www.karger.com/aha

Karger $\stackrel{\text { ' }}{5}$

BOPEN ACCESS
(C) 2022 The Author(s)

Published by S. Karger AG, Basel

This is an Open Access article licensed under the Creative Commons Attribution-NonCommercial-4.0 International License (CC BY-NC) (http://www.karger.com/Services/OpenAccessLicense), applicable to the online version of the article only. Usage and distribution for commercial purposes requires written permission.
$=0.0030$ ). Among the 45 patients who responded to splenectomy, $51.1 \%$ relapsed subsequently, and a significantly lower relapse rate was noted in the stable IVIG responders $(12.5 \%, p=0.0220)$ than in nonresponders $(60.0 \%)$ and poor responders (59.4\%). Conclusions: First-line IVIG response is indicated as a useful predictive factor for response to splenectomy.

(C) 2022 The Author(s)

Published by S. Karger AG, Basel

\section{Introduction}

Immune thrombocytopenia (ITP) is an immune disease characterized by thrombocytopenia (platelet [PLT] counts of $<100 \times 10^{9} / \mathrm{L}$ ) and associated bleeding symptoms [1]. Steroid, intravenous immunoglobulin (IVIG), anti-D immunoglobulin, and several immunosuppressive agents are used for the primary treatment of ITP. However, a significant number of patients do not respond to first-line therapy or relapse after an initial response [1-3]. As a second-line therapy for refractory or relapsed ITP, splenectomy is still an option, although thrombopoietin receptor agonists (TPO-RA) and other novel agents are widely introduced these days [4]. Splenectomy is con-
Correspondence to:

Jae-Ho Yoon, royoon@catholic.ac.kr 
sidered safe for young patients without comorbidity, but the older patients or patients with several comorbid conditions are known to have a higher risk of postsplenectomy complications, hence the choice of surgery in these patients should be cautiously decided [5-7]. An indicator that can reliably predict the efficacy of splenectomy is still being explored $[4,8]$.

IVIG is a widely used first-line agent for ITP [9]. It has a rapid-acting time of fewer than 3 days, and its duration of action is no longer than 4 weeks [3]. Therefore, we can easily evaluate its response quickly and independently. In addition, the role of the spleen in ITP pathophysiology overlaps the mechanism of action of IVIG $[10,11]$, and the direct action of IVIG on the spleen has also been reported [12]. In this respect, we tried to identify a possible predictive role of IVIG in determining the outcome of splenectomy in patients with relapsed or refractory ITP.

\section{Methods}

\section{Patients and Definitions}

We retrospectively investigated the data of 52 consecutive patients who underwent splenectomy as a second-line treatment for ITP between 2009 and 2019. The earliest ITP diagnosis was established in March 1990, and the most recent patient was diagnosed in June 2018. The range of follow-up period for all patients was 7.8-291.5 months after diagnosis and 1.6-128.4 months after splenectomy. All patients were comprehensively assessed for possible causes of thrombocytopenia other than ITP, and bone marrow aspiration and biopsy were performed in patients older than 50 years of age or refractory to first-line therapy. This study was approved by Institutional Review Board (KC21RISI0129) and was conducted in accordance with the Declaration of Helsinki. Informed consent was obtained from all individual participants included in the study.

Treatment response was evaluated according to the International Working Group criteria: PLT counts $\geq 30 \times 10^{9} / \mathrm{L}$, twice increase from the baseline PLT counts, and absence of bleeding was regarded as a response [13]. Relapse was diagnosed when a patient failed to meet the response criteria. All patients in our data were diagnosed as timely persistent or chronic ITP according to the International Working Group criteria [13]. Further, we diagnosed a patient who never met the response criteria during the first-line treatment strategy with primary refractory ITP.

We classified patients according to their response to first-line IVIG therapy. In patients who were repeatedly administered IVIG during the first-line treatment period, the last response was used as the criterion for evaluating the IVIG response. Patients who did not show response to IVIG at all were classified as nonresponders; patients who responded to IVIG but failed to maintain PLT response for longer than 4 weeks were classified as poor responders; and patients who responded well to IVIG and maintained a response for longer than 4 weeks were classified as stable responders. Stable responders also relapsed subsequently, and the PLT level was maintained through repeated IVIG administration or another first-line therapy. The patient selection and classification processes are summarized in Figure 1.

\section{Processes of ITP Treatment}

When a patient was newly diagnosed with ITP, methylprednisolone pulse therapy of higher than $2 \mathrm{mg} / \mathrm{kg} /$ day was administered as a first-line therapy, if not contraindicated, and tapered off for over 4 weeks. If initial steroid response was not observed with elevation of PLT count higher than $30 \times 10^{9} / \mathrm{L}$ or twice increase from the baseline level at diagnosis, IVIG $1 \mathrm{~g} / \mathrm{kg}$ for 2 days (total $2 \mathrm{~g} / \mathrm{kg}$ ) or anti-D $250 \mathrm{IU} / \mathrm{kg}$ for 2 days (total $500 \mathrm{IU} / \mathrm{kg}$ ) was administered. In patients who did not respond to IVIG or anti-D immunoglobulin, we considered additional immunosuppressive agents, such as azathioprine, danazol, methotrexate, vincristine, and mycophenolate mofetil. In case of patients responding to IVIG, we administered IVIG repeatedly at intervals of at least 3 weeks when the PLT count decreased to less than $30 \times 10^{9} / \mathrm{L}$. In patients with relapsed or refractory ITP, rituximab, splenectomy, or TPO-RA were considered as second-line treatments. All patients in our data underwent splenectomy, and the timing of splenectomy was determined by the clinician by weighing the risks and benefits of surgery and patient preferences. Therefore, patients underwent splenectomy when relapse/refractory ITP was confirmed or after attempting sufficient other medical therapies. Open or laparoscopic surgery was performed, and regardless of the type of surgery, we intended to increase the PLT counts higher than $50 \times 10^{9} / \mathrm{L}$ just before splenectomy by means of IVIG or PLT transfusion. If the PLT count did not reach the target level, the PLTs were additionally transfused during the surgery. The response to splenectomy was checked by measuring the PLT count daily during admission, and weekly follow-up was done after discharge. We defined the postsplenectomy response if the patient's PLT count was maintained higher than $50 \times 109 / \mathrm{L}$ until 4 weeks after splenectomy. Salvage treatment was considered for patients who were refractory to splenectomy or relapsed after response to splenectomy.

\section{Statistical Analysis}

We investigated the response rate, relapse rate, relapse-free survival (RFS), and postoperative complications after splenectomy, and the prognostic significance was analyzed according to the response to first-line IVIG therapy. The treatment outcomes after salvage therapy were also identified. Each variable was described using a number with percentage or median with a range. The distribution of categorized variables according to IVIG response groups was compared using the $\chi^{2}$ test and Fisher's exact test. Continuous variables were tested by the Kruskal-Wallis test or Wilcoxon rank-sum test. RFS was estimated using the Kaplan-Meier estimate and 95\% confidence interval (CI), calculating the event date as the day when refractoriness or relapse was confirmed. There was no problem while using this estimate as none of the patients experienced competing risks, such as death, before the occurrence of relapse. The log-rank test was used to compare RFS between the groups. All statistical analyses were performed using the $\mathrm{R}$ software (version 4.0.3). A $p$ value of $<0.05$ was considered statistically significant. All $p$ values were two-sided. 


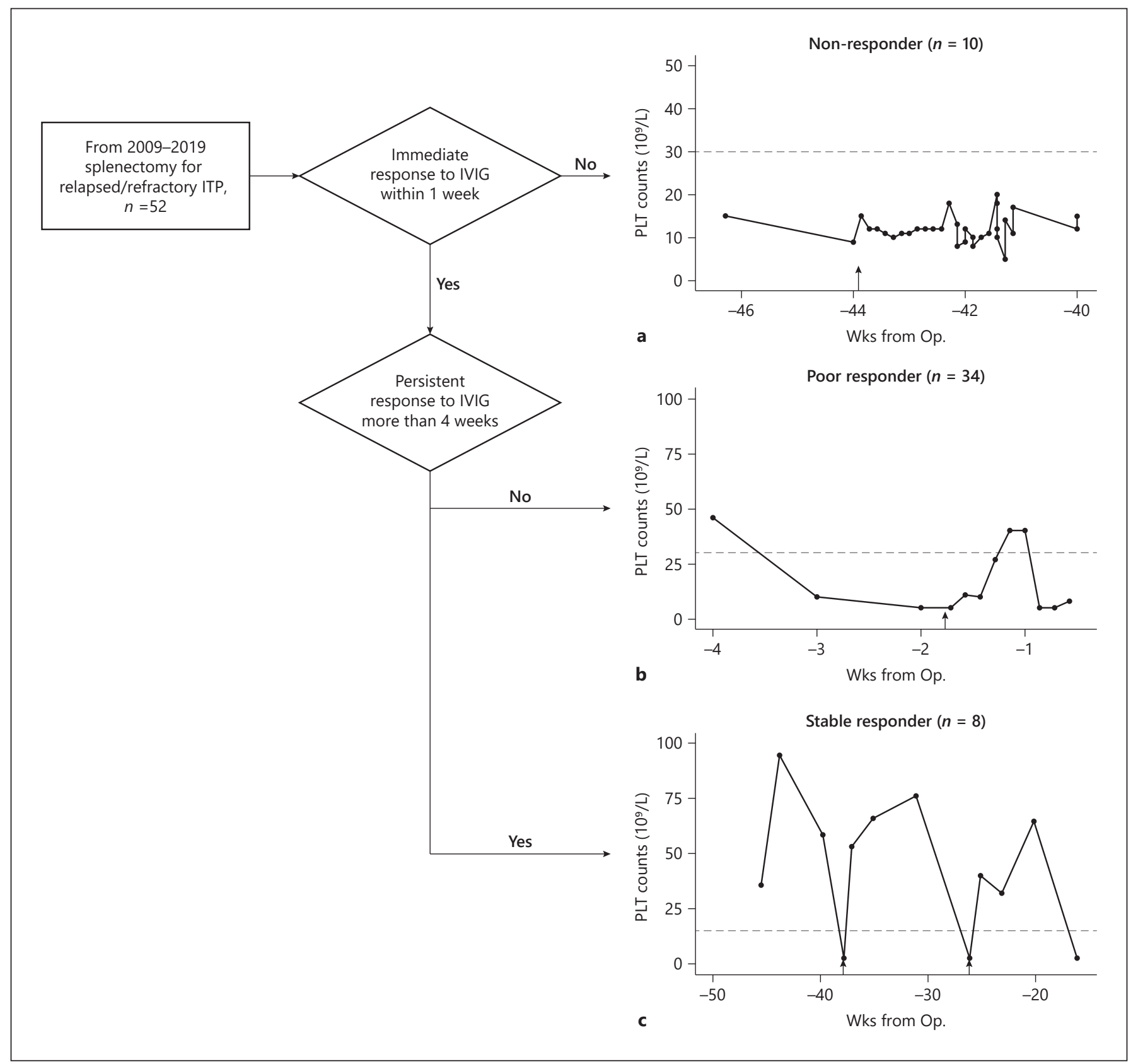

Fig. 1. a-c Patient selection and classification processes. Three PLT flow graphs are the real example of each group from our data. Dashed line indicates the PLT counts of $30 \times 10^{9} / \mathrm{L}$. Arrows are the timing of IVIG administration.

\section{Results}

\section{Baseline Characteristics}

The demographics and baseline characteristics of the patients are listed in Table 1. Patients were divided into three subgroups according to IVIG response: nonresponders $(n=10)$, poor responders $(n=34)$, and stable responders $(n=8)$. Patients had a median age of 38 years (range: $25-72$ years), 40 (76.9\%) patients were $<55$ years of age, and $15(28.8 \%)$ patients were men. Regarding etiologies, 19 (36.5\%) patients had primary ITP, 15 (28.8\%) patients had concomitant immune disorders, and 18 (34.6\%) had undetermined etiology. The median time between ITP diagnosis and splenectomy was 25.9 months 
Table 1. Baseline characteristics of ITP patients treated with splenectomy according to IVGV response

\begin{tabular}{|c|c|c|c|c|c|}
\hline Age, median (range), $n$ (\%) & $38(25-72)$ & $36(26-60)$ & $42.5(25-72)$ & $32(25-57)$ & 0.3220 \\
\hline$\geq 55$ & $12(23.1)$ & $2(20.0)$ & $9(26.5)$ & $1(12.5)$ & \\
\hline \multicolumn{6}{|l|}{ Gender, $n(\%)$} \\
\hline Male & $15(28.8)$ & $3(30.0)$ & $9(26.5)$ & $3(37.5)$ & 0.8212 \\
\hline Primary & $19(36.5)$ & $4(40.0)$ & $12(35.3)$ & $3(37.5)$ & 0.6977 \\
\hline Secondary & $15(28.8)$ & $4(40.0)$ & $10(29.4)$ & $1(12.5)$ & \\
\hline Undetermined & $18(34.6)$ & $2(20.0)$ & $12(35.3)$ & $4(50.0)$ & \\
\hline Time to splenectomy, months median (range), $n$ (\%) & $25.9(1.4-284.1)$ & $13.5(1.6-45.8)$ & $28.3(1.5-284.1)$ & $12.6(1.4-263.7)$ & 0.2884 \\
\hline$<12$ months & $18(34.6)$ & $4(40.0)$ & $10(29.4)$ & $4(50.0)$ & \\
\hline$\geq 4$ & $6(11.5)$ & $1(10.0)$ & $3(8.8)$ & $2(25.0)$ & \\
\hline \multicolumn{6}{|l|}{ Concomitant immunosuppressive therapies } \\
\hline Danazol & $6(11.5)$ & 0 & $5(14.7)$ & $1(12.5)$ & 0.6811 \\
\hline Mycophenolate mofetil & $1(1.9)$ & $1(10.0)$ & 0 & 0 & 0.3462 \\
\hline Azathioprine & $5(9.6)$ & $2(20.0)$ & $2(5.9)$ & $1(12.5)$ & 0.8570 \\
\hline Methotrexate & $1(1.9)$ & $1(10.0)$ & 0 & 0 & 0.3462 \\
\hline Vincristine & $2(3.8)$ & 0 & $1(2.9)$ & $1(12.5)$ & 0.3205 \\
\hline \multicolumn{6}{|l|}{ Minimal PLT count, $n(\%)$} \\
\hline$<5 \times 10^{9} / \mathrm{L}$ & $31(59.6)$ & $7(70.0)$ & $24(70.6)$ & $0(0.0)$ & $<0.0001$ \\
\hline $5-30 \times 10^{9} / \mathrm{L}$ & $21(40.4)$ & $3(30.0)$ & $10(29.4)$ & $8(100.0)$ & \\
\hline \multicolumn{6}{|l|}{ Vaccination, $n(\%)$} \\
\hline S. pneumoniae & $50(96.2)$ & $9(90.0)$ & $33(97.1)$ & $8(100.0)$ & 0.5769 \\
\hline
\end{tabular}

${ }^{\dagger}$ Steroid, IVIG, anti-D immunoglobulin, TPO-RA, and rituximab were counted as lines of treatments. Other concomitant therapeutic agents were not counted as the lines of treatment.

(range: $1.4-284.1$ months) and 18 (34.6\%) patients underwent splenectomy within 12 months. All the patients in our study were treated with at least two therapeutic agents; $31(59.6 \%)$ patients were treated with two therapies (steroid and IVIG), $15(28.8 \%)$ patients were treated with three therapies, and $6(11.5 \%)$ patients were treated with four or more therapeutic agents. During medical therapies including IVGV, all patients showed relapse or refractoriness with reduced PLT counts to less than $30 \times$ $10^{9} / \mathrm{L}-31(59.6 \%)$ of them had minimal PLT counts of 5 $\times 10^{9} / \mathrm{L}$ or less. Most patients were vaccinated against Streptococcus pneumoniae and Haemophilus influenzae (96.2\% and $86.5 \%$, respectively), whereas approximately half of the patients $(57.7 \%)$ were vaccinated against Neisseria meningitidis. The baseline characteristics according to IVIG response were similar among the three subgroups, except for minimal PLT counts during medical therapy. The proportion of minimal PLT counts $(\leq 5 \times$ $10^{9} / \mathrm{L}$ ) was found in $70.0 \%$ and $70.6 \%$ of the IVIG nonresponders and poor responders, respectively, and all the IVIG stable responders had minimal PLT counts of $>5 \times$ $10^{9} / \mathrm{L}$.

\section{Response after Splenectomy}

Splenectomy was performed between 2009 and 2014 in $61.5 \%$ of patients and between 2015 and 2019 in 38.5\% of patients (Table 2). All patients underwent laparoscopic splenectomy except one in 2017. For safe splenectomy, we applied IVGV in previous responders and performed transfusion to rapidly increase the PLT count as high as 
Table 2. Splenectomy outcomes of patients

\begin{tabular}{|c|c|c|c|c|c|}
\hline Variables & $\begin{array}{l}\text { Total } \\
(N=52)\end{array}$ & $\begin{array}{l}\text { Nonresponder } \\
(N=10)\end{array}$ & $\begin{array}{l}\text { Poor responder } \\
(N=34)\end{array}$ & $\begin{array}{l}\text { Stable responder } \\
(N=8)\end{array}$ & $p$ value \\
\hline \multicolumn{6}{|l|}{ Year of splenectomy, $n$ (\%) } \\
\hline 2015-2019 & $20(38.5)$ & $5(50.0)$ & $12(35.3)$ & $3(37.5)$ & \\
\hline \multicolumn{6}{|l|}{ Type of op., $n$ (\%) } \\
\hline Open & $1(1.9)$ & $1(10.0)$ & $0(0.0)$ & $0(0.0)$ & 0.3462 \\
\hline \multicolumn{6}{|l|}{ 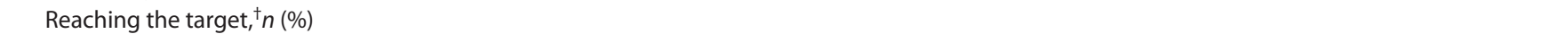 } \\
\hline No & $10(19.2)$ & $8(80.0)$ & $2(5.9)$ & $0(0.0)$ & $<0.0001$ \\
\hline Yes & $42(80.8)$ & $2(20.0)$ & $32(94.1)$ & $8(100.0)$ & \\
\hline \multicolumn{6}{|l|}{ Response, $n(\%)$} \\
\hline No response & $7(13.5)$ & $5(50.0)$ & $2(5.9)$ & $0(0.0)$ & 0.0030 \\
\hline RFS, weeks (median, 95\% Cl) & $137(10.4-350.0)$ & $4.6\left(0.7-\mathrm{NA}^{\ddagger}\right)$ & $118.4(9.0-350.0)$ & $N A^{\ddagger}$ & 0.0439 \\
\hline 2-year relapse-free rate, $\%(95 \% \mathrm{Cl})$ & $55.6(43.6-70.9)$ & $40.0(18.7-95.5)$ & $52.9(38.6-72.7)$ & $87.5(67.3-100)$ & \\
\hline PLT at post op $1 \mathrm{wk}, 10^{9} / \mathrm{L}$ (median, range) & $257(9-1,152)$ & $114.5(9-600)$ & $293(19-960)$ & $393.5(115-1,152)$ & 0.0392 \\
\hline \multicolumn{6}{|l|}{ Complications } \\
\hline Ischemic heart disease & $1(1.9)$ & $0(0.0)$ & $1(2.9)$ & $0(0.0)$ & 1.000 \\
\hline Venous thrombosis & $3(5.8)$ & $1(10.0)$ & $2(5.9)$ & $0(0.0)$ & 0.7292 \\
\hline Sepsis & $1(1.9)$ & $0(0.0)$ & $1(2.9)$ & $0(0.0)$ & 1.000 \\
\hline Others ${ }^{\S}$ & $2(3.8)$ & $0(0.0)$ & $2(5.9)$ & $0(0.0)$ & 1.000 \\
\hline Death, $n$ (\%) & $4(3.8)$ & $2(20.0)$ & $2(5.9)$ & $0(0.0)$ & 0.2651 \\
\hline
\end{tabular}
hernia.

op., operation; NA, not available; wk, week. ${ }^{\dagger} 50 \times 10^{9} / \mathrm{L}$. ${ }^{\ddagger}$ Not available because not enough people have experienced the event to estimate. ${ }^{\S}$ Incision site

we could. Hence, the patients had a median PLT count of $85 \times 109 / \mathrm{L}$ (range: $5-352 \times 109 / \mathrm{L}$ ) just before splenectomy; according to IVIG response, a median PLT count was $41.5 \times 109 / \mathrm{L}$ (range: $5-102 \times 109 / \mathrm{L}$ ) in nonresponders, $93.5 \times 109 / \mathrm{L}$ (range: $31-352 \times 109 / \mathrm{L}$ ) in poor responders, and $123.5 \times 109 / \mathrm{L}$ (range: $57-241 \times 109 / \mathrm{L}$ ) in stable responders $(p=0.0004)$. The proportion of patients who reached the target level of PLT count before splenectomy $\left(\geq 50 \times 10^{9} / \mathrm{L}\right)$ was $20.0 \%$ in IVIG nonresponders, $94.1 \%$ in poor responders, and $100 \%$ in stable responders $(p<$ 0.0001). Overall, $45(86.5 \%)$ patients responded to splenectomy, and the response rates among the IVIG responder subgroups were significantly different: $50 \%$ in nonresponders, $94.1 \%$ in poor responders, and $100 \%$ in stable responders $(p=0.0030$, among all the subgroups; $p$ $=0.0017$, between nonresponders and other subgroups). Among the 45 patients who responded to splenectomy, $23(51.1 \%)$ relapsed after a median period of 31.5 months (95\% CI: 2.4-80.7), including 1 (12.5\%) stable responder, 19 (59.4\%) poor responders, and $3(60.0 \%)$ nonre- sponders; the relapse rates according to IVIG response showed a significant difference $(p=0.0683$, among all the groups; $p=0.0220$, between stable responders and the other subgroups). The median RFS was 4.6 weeks in IVIG nonresponders and 118.4 weeks in poor responders, while that of stable responders did not reach the median RFS ( $p=0.0439$ ) (Fig. 2a). The estimated 2-year RFS in the three subgroups was $40.0 \%$ (95\% CI: 18.7-95.5) in nonresponders, $52.9 \%$ (95\% CI: $38.6-72.7$ ) in poor responders, and $87.5 \%$ (95\% CI: 67.3-100) in stable responders. When follow-up PLT counts of patients were investigated, differences according to IVIG response were observed. We identified the trends of increase in PLT counts 1 week after splenectomy as a better response to IVIG therapy (median [range] PLT counts: $114.5 \times$ $10^{9} / \mathrm{L}\left[9-600 \times 10^{9} / \mathrm{L}\right], 293.0 \times 10^{9} / \mathrm{L}\left[19-960 \times 10^{9} / \mathrm{L}\right]$, and $393.5 \times 10^{9} / \mathrm{L}\left[115-1,152 \times 10^{9} / \mathrm{L}\right]$ in nonresponders, poor responders, and stable responders, respectively, $p=$ 0.0392; Fig. 2b). 


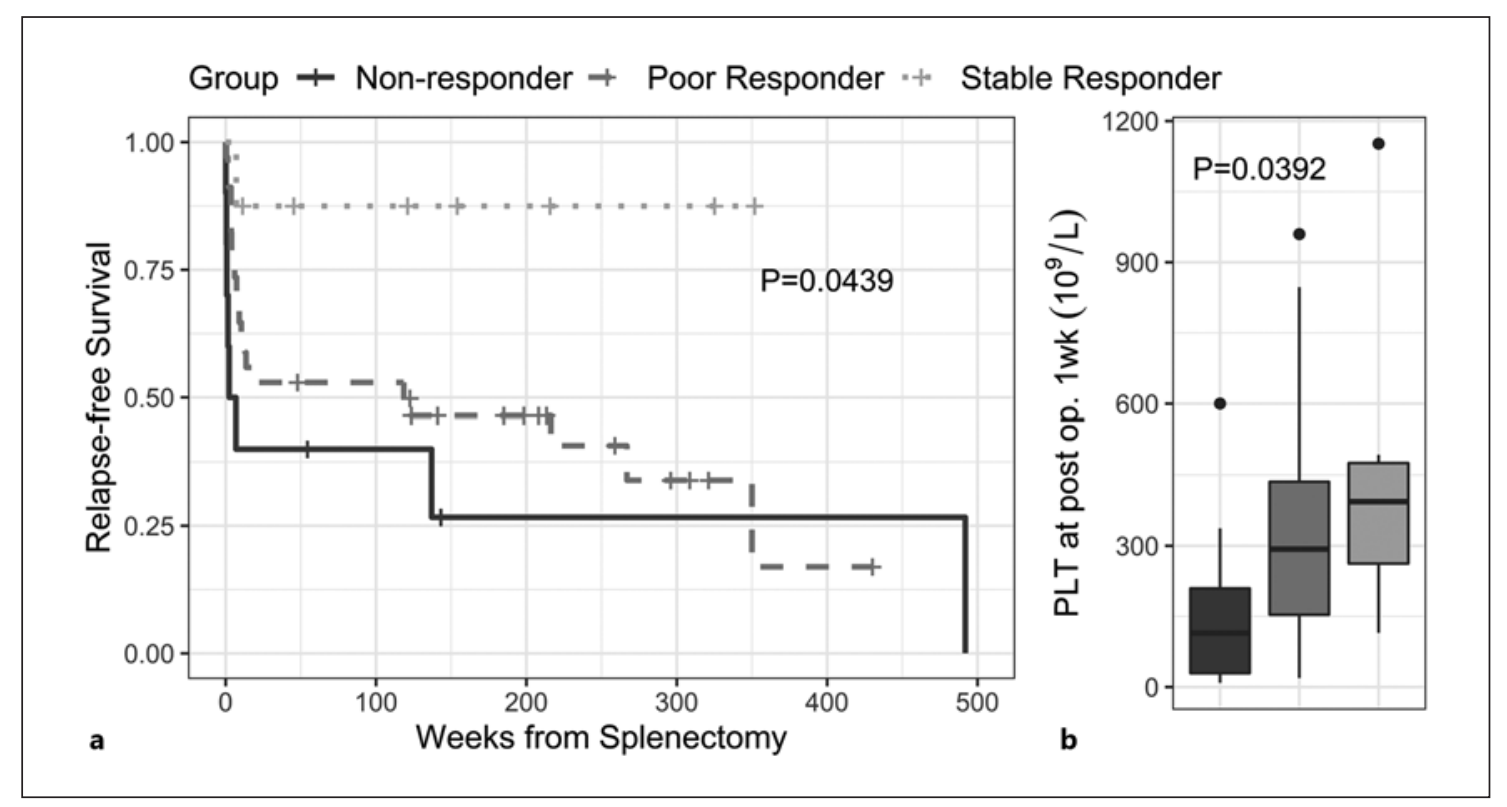

Fig. 2. RFS and PLT follow-up after splenectomy, according to IVIG response groups. a Kaplan-Meier curves of RFS of three IVIG response groups. b Boxplots of PLT counts after 1 week from splenectomy of three IVIG response groups. Points in plots are outliers, which are larger than $1.5 \times$ interquartile range (distance between the first and third quartiles) from the 75th percentiles.

\section{Complications after Splenectomy}

The following postsplenectomy complications were observed: 1 case of ischemic heart disease, 3 cases of venous thrombosis ( 1 case of splenic vein thrombosis, 1 case of portal vein thrombosis, and 1 case of portal vein thrombosis with pulmonary thromboembolism), 1 case of sepsis, and 2 cases of incision site hernia. Most of these complications were safely managed. Four deaths occurred during the follow-up period, and all of these patients died after ITP relapse: 1 patient was refractory to splenectomy and died owning to severe gastrointestinal bleeding at 6 months after splenectomy; another patient underwent coronary artery bypass graft surgery 2 months after splenectomy owning to aggravation of ischemic heart disease and died of postoperative infection; another patient died 4 years after splenectomy due to end-stage renal disease that was diagnosed before splenectomy; the other died of diffuse large B-cell lymphoma at 3 years after splenectomy.

\section{Salvage Treatments for Postsplenectomy Relapse}

Overall, there were 30 patients ( 7 refractory and 23 relapsed after splenectomy) who were candidates for salvage treatments: one IVIG stable responders; 21 IVIG poor responders; and 8 nonresponders before splenec- tomy. The previously stable IVIG responders who relapsed after splenectomy maintained a low, but stable PLT count without specific salvage therapy. We presented clinical courses in 8 nonresponders and 21 poor responders after splenectomy (Table 3). The interval between splenectomy and salvage treatment was not significantly different between IVIG nonresponders and poor responders (6.5 weeks and 12.9 weeks, respectively, $p=$ 0.4501).

Various salvage regimens were tried, except for 3 poor responders who were treated with supportive care only. Overall, 21 (80.8\%) of 26 patients who received salvage treatment showed a response again, and 7 (26.9\%) subsequently stopped all medications after a median followup duration of 32.7 months (range: 0.4-114.2 months). Among the patients who showed a response to salvage treatment, only 1 patient lost response again at the last follow-up. Response to retreatment, the rate at which the drug could be stopped, and final disease status were similar between poor responders and nonresponders. After salvage treatment, the follow-up timing of PLT counts was different for each patient; however, a higher PLT count was observed in poor responders than in nonresponders (Fig. 3). When we compared the PLT counts at the last time when the PLTs of the patients were evenly 
Fig. 3. PLT follow-up after salvage treatment in postsplenectomy refractory/relapsed patients, grouped by IVIG response groups (nonresponse or poor response). The vertical lines indicate interquartile range (distance between the first and third quartiles). The number of examined patients shows the number of patients whose PLT counts were examined at each month. ${ }^{*} p$ value is estimated from the PLT counts after 3 months from salvage treatment.

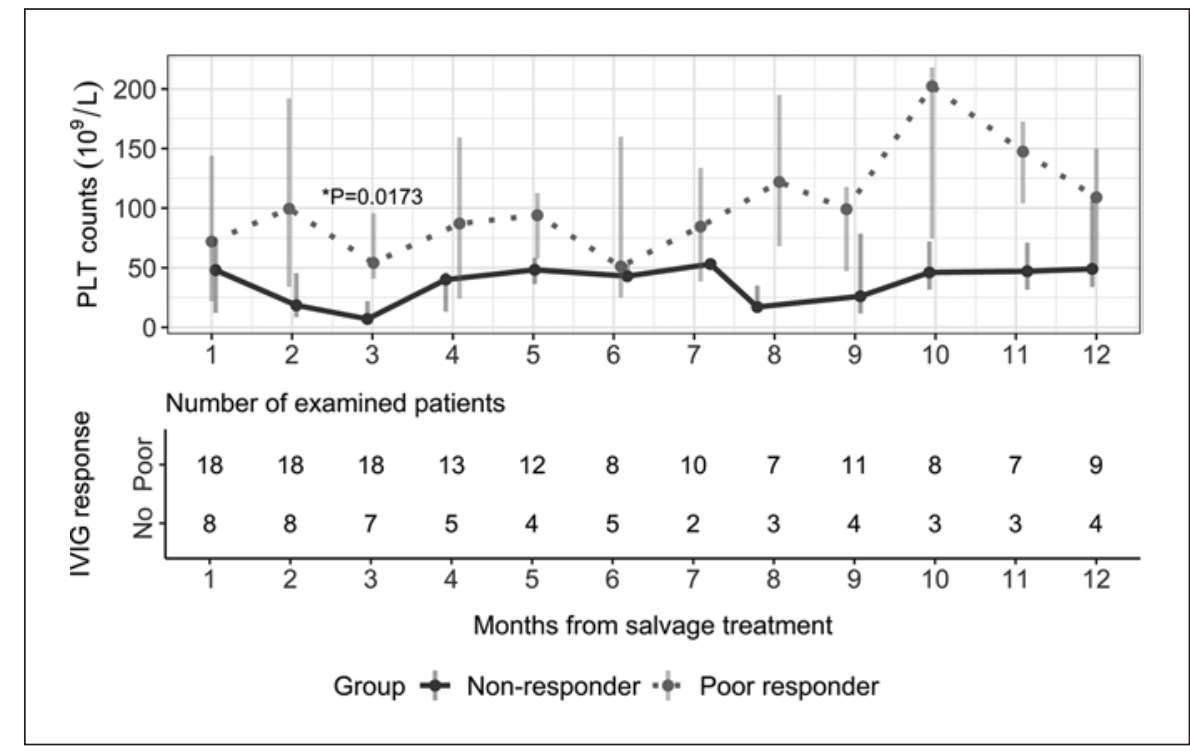

Table 3. Salvage treatment outcomes of refractory/relapsed patients

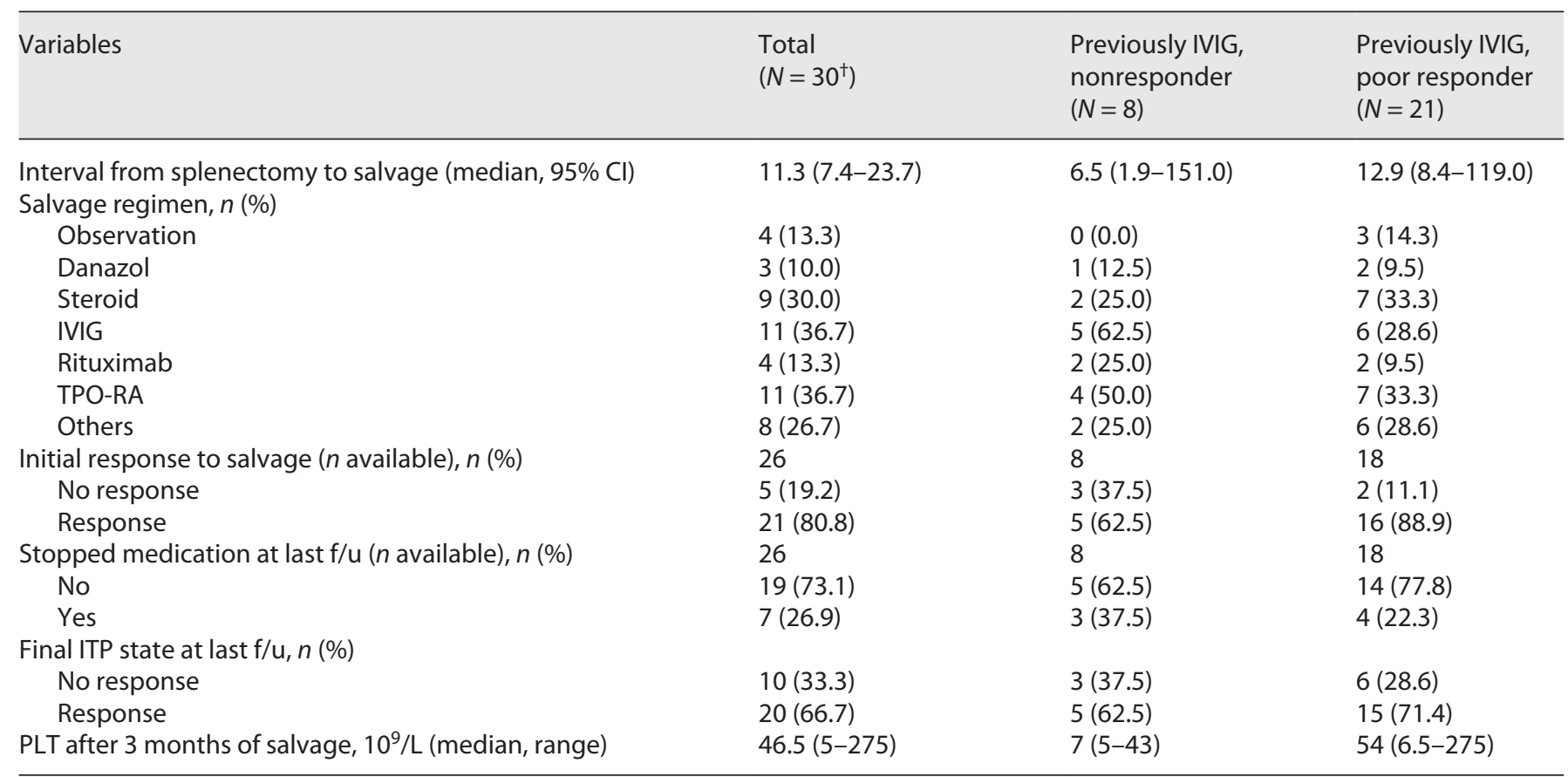

$\mathrm{f} / \mathrm{u}$, follow-up; $N$, number. 'The number of patients who were previously IVIG stable responder was only one, so the results of previously IVIG stable-responder group were not described as distinct column.

tracked (at 3 months after salvage), the median PLT count was $7 \times 10^{9} / \mathrm{L}$ (range: $5-43 \times 10^{9} / \mathrm{L}$ ) in nonresponders and $54 \times 10^{9} / \mathrm{L}$ (range: $6.5-575 \times 10^{9} / \mathrm{L}$ ) in poor responders, the difference being statistically significant $(p=0.0173)$.

Splenectomy Outcomes by IVIG Response

\section{Discussion}

Splenectomy is considered a second-line treatment for patients with relapsed or refractory chronic ITP, as the spleen is quite involved in the destruction of platelets [4]. 
However, splenectomy is one of the major abdominal surgeries, and patients with ITP cannot avoid the risk of bleeding because of thrombocytopenia [14]. In addition, the risks of infection and thrombosis must also be considered $[14,15]$. With the emergence of novel agents, such as TPO-RA, the tendency to avoid splenectomy is increasing and recent guidelines indicate delaying splenectomy for at least 12 months after diagnosis, especially in patients who are reluctant to undergo surgery [2]. Nevertheless, splenectomy is still considered a second-line treatment in relapsed or refractory ITP especially for patients who cannot afford to take TPO-RA due to side effects or refuse the long-term therapy due to financial or medical problems. In some countries, unfortunately, TPO-RA is approved for a third-line treatment after failure to splenectomy. Therefore, it is still important to accurately evaluate the benefits and risks of splenectomy.

This study showed that the response to IVIG - which is commonly used as a first-line treatment for ITP with rapid response evaluation - was useful in predicting outcomes of splenectomy. However, the patient's PLT response during medical treatment was not entirely attributable to IVIG because most of the patients were treated with other agents concomitantly. Thus, all enrolled patients in this study were refractory to IVGV or relapsed eventually even though they showed response to IVGV with concomitant therapeutic agents. Stable IVGV responders eventually relapsed despite concomitant immunotherapeutic agents and were treated with IVGV repeatedly. Therefore, we do not insist the response was solely due to IVIG, but we regard IVGV as the fastest way to check the degree of refractoriness and could be a good guide to the next treatment plan. We finally suggest that IVIG responsiveness can be considered a practical prognostic indicator for splenectomy.

Responses to IVIG and their associations with splenectomy outcomes were analyzed in a few previous studies. However, the results were conflicting, and no studies could provide strong evidence [16-21]. Besides, previous studies were conducted based on the dichotomous classification of whether the IVIG response existed or not. We classified patients into nonresponders, poor responders, and stable responders by analyzing both immediate response to IVIG therapy and the maintenance of the response state after 4 weeks altogether, and these three groups were found to have distinct features.

Responsiveness to IVIG reflects the pathophysiology of ITP, which can be effectively treated by splenectomy. The most well-known mechanism of action of IVIG is the inhibition of $\mathrm{Fc}$ gamma receptor $(\mathrm{Fc} \gamma \mathrm{R})$-mediated PLT destruction, which is achieved by the saturation of $\mathrm{F} c \gamma \mathrm{R}$ [10], and splenic macrophages play a key role in Fc $\gamma \mathrm{R}$ mediated phagocytosis [11]. In addition, the spleen promotes the production of antiplatelet autoantibodies [22], and elimination of the antibodies can be accelerated by IVIG [10]. The direct action of IVIG on the spleen was also reported that the proportion of myeloid-derived suppressor cells in the population of splenocytes increased with direct exposure to IVIG in patients with ITP [12]. Therefore, responses to IVIG and splenectomy were symmetric; poor responses after splenectomy in IVIG nonresponders, transient response in poor IVIG responders, and good and sustained response in stable IVIG responders. Mechanisms other than spleen-associated pathways, such as impaired megakaryocytopoiesis or T-cell mediated PLT destruction, may work commonly in non- or poor IVIG responders $[23,24]$. On the other hand, we observed that the PLT response was different between nonresponders and poor responders after splenectomy, which may suggest the differences in pathophysiology other than the spleen-associated pathway exist between the two groups. For example, the expression of anti-glycoprotein Ib/IX autoantibodies is a distinguishing characteristic between IVIG nonresponders and responders in adult patients with ITP [25]. Further research on IVIG response and ITP pathophysiology is warranted.

Our data indicated that $86.5 \%$ of patients with relapsed or refractory ITP showed a response after second-line splenectomy. However, $51.1 \%$ of patients relapsed subsequently, which was higher than other previous data from the same region (29.1\% in Park et al. [7] and 35.0\% in Han et al. [26]). The higher relapse rate may be because of the difference in patient registration time of each study. In the previous studies, splenectomy was often considered earlier [8], whereas our study included patients for whom surgical therapy was delayed as much as possible after using repetitive medical therapies [6]. This fact can be seen by the number of treatments tried before surgery in our study patients (none of the patients tried only one agent for treatment before surgery and $40.4 \%$ tried more than three agents), compared to other studies (15.8\% of patients in Park et al. [7] tried just steroids before splenectomy and only $28.8 \%$ tried more than three therapeutic agents and no mention of the number of treatments in Han et al. [26]). Differences in the timing of response evaluation may also have contributed to the variances in relapse rates, yet there is no standard timing of judgment to evaluate the response of patients to splenectomy [7]. Additionally, our study consisted of a high proportion of patients with secondary ITP $(15 / 52,28.8 \%$, 5 with an- 
tiphospholipid syndrome, 1 with systemic lupus erythematosus, and 9 with Evans syndrome), and the high relapse rates could be partly attributed to this feature. It was difficult to draw definitive conclusions from our data because of the large number of cases with undetermined etiology, and few studies have revealed a link between the underlying disease and relapse after splenectomy [4].

As presented in our data, the efficacy of splenectomy in patients who showed no or poor response to IVIG was dismal. Furthermore, maintaining a sufficient PLT count before surgery is thought to be important to reduce perioperative surgical complications [27]. In this respect, the surgical risk is considered high in IVIG nonresponders, as our data showed that a high proportion $(80.0 \%)$ of IVIG nonresponders was not able to reach a sufficient PLT count before splenectomy. Hence, especially for patients with no or poor IVIG response, it should be considered to treat them initially with medical agents as a second-line therapy, not considering the surgery.

This study has several limitations. Since our study did not include a sufficient number of patients and we categorized the patients into three subgroups, statistical power might not be adequate and adjusting the effects of other variables, such as multivariate modeling, was unavailable. However, there were enough factors that showed significance, and no significant difference in baseline characteristics was observed among the IVIG response groups.

In conclusion, our data showed that the response to first-line IVIG therapy could be a predictive factor for response and relapse after splenectomy. Therefore, other treatment options, such as TPO-RA or other medical therapies, should be considered prior to splenectomy in patients showing poor or no response to IVIG. Further clinical trials in larger patient cohorts are needed to validate our findings.

\section{Statement of Ethics}

This study was approved by Institutional Review Board (KC21RISI0129) and was conducted in accordance with the Declaration of Helsinki. Informed consent was obtained from all individual participants included in the study.

\section{Conflict of Interest Statement}

The authors report no conflict of interest.

\section{Funding Sources}

No funding sources for this work.

\section{Author Contributions}

Conception and design: D. Kwag, J.-H. Yoon. Development of methodology: D. Kwag, G.J. Min, and J.-H. Yoon. Data acquisition: S.-S. Park, S. Park, S.-E. Lee, J.-H. Yoon, B.-S. Cho, K.-S. Eom, Y.-J. Kim, H.-J. Kim, S. Lee, C.-K. Min, S.-G. Cho, D.-W. Kim, and J.W. Lee. Analysis and interpretation of data: D. Kwag, S.-S. Park, J.-H. Yoon, B.-S. Cho, H.-J. Kim, and J.W. Lee. Writing, review, and/or revision of the manuscript: D. Kwag, J.-H. Yoon, S.-E. Lee, D.-W. Kim, and J.W. Lee. Administrative, technical, or material support: S.-S. Park, S. Park, S.-E. Lee, K.-S. Eom, and Y.-J. Kim. Study supervision: J.-H. Yoon.

\section{Data Availability Statement}

For the original data, please contact the corresponding author (royoon@catholic.ac.kr).

\section{References}

1 Lambert MP, Gernsheimer TB. Clinical updates in adult immune thrombocytopenia. Blood. 2017;129:2829-35.

2 Neunert C, Terrell DR, Arnold DM, Buchanan G, Cines DB, Cooper N, et al. American Society of Hematology 2019 guidelines for immune thrombocytopenia. Blood Adv. 2019;3:3829-66.

3 Khan AM, Mydra H, Nevarez A. Clinical practice updates in the management of immune thrombocytopenia. P T. 2017;42:75663.

4 Chaturvedi S, Arnold DM, McCrae KR. Splenectomy for immune thrombocytopenia: down but not out. Blood. 2018;131:1172-82.

Splenectomy Outcomes by IVIG Response
5 Cortelazzo S, Finazzi G, Buelli M, Molteni A, Viero P, Barbui T. High risk of severe bleeding in aged patients with chronic idiopathic thrombocytopenic purpura. Blood. 1991;77: 31-3.

6 Gonzalez-Porras JR, Escalante F, Pardal E, Sierra M, Garcia-Frade LJ, Redondo S, et al. Safety and efficacy of splenectomy in over 65 -yrs-old patients with immune thrombocytopenia. Eur J Haematol. 2013;91:236-41.

7 Park YH, Yi HG, Kim CS, Hong J, Park J, Lee $\mathrm{JH}$, et al. Clinical outcome and predictive factors in the response to splenectomy in elderly patients with primary immune thrombocytopenia: a multicenter retrospective study. Acta Haematol. 2016;135(3):162-71.
8 Kojouri K, Vesely SK, Terrell DR, George JN Splenectomy for adult patients with idiopathic thrombocytopenic purpura: a systematic review to assess long-term platelet count responses, prediction of response, and surgical complications. Blood. 2004;104:2623-34.

9 Neunert C, Lim W, Crowther M, Cohen A, Solberg L, Crowther MA. The American Society of Hematology 2011 evidence-based practice guideline for immune thrombocytopenia. Blood. 2011;117:4190-207.

10 Jin F, Balthasar JP. Mechanisms of intravenous immunoglobulin action in immune thrombocytopenic purpura. Hum Immunol. 2005;66:403-10. 
11 Audia S, Santegoets K, Laarhoven AG, Vidarsson G, Facy O, Ortega-Deballon P, et al. $\mathrm{Fc} \gamma$ receptor expression on splenic macrophages in adult immune thrombocytopenia. Clin Exp Immunol. 2017;188:275-82.

12 Aslam R, Burack WR, Segel GB, McVey M, Spence SA, Semple JW. Intravenous immunoglobulin treatment of spleen cells from patients with immune thrombocytopenia significantly increases the percentage of myeloid-derived suppressor cells. Br J Haematol. 2018;181:262-4.

13 Rodeghiero F, Stasi R, Gernsheimer T, Michel M, Provan D, Arnold DM, et al. Standardization of terminology, definitions and outcome criteria in immune thrombocytopenic purpura of adults and children: report from an international working group. Blood. 2009;113: 2386-93.

14 Portielje JE, Westendorp RG, Kluin-Nelemans HC, Brand A. Morbidity and mortality in adults with idiopathic thrombocytopenic purpura. Blood. 2001;97:2549-54.

15 Vianelli N, Palandri F, Polverelli N, Stasi R, Joelsson J, Johansson E, et al. Splenectomy as a curative treatment for immune thrombocytopenia: a retrospective analysis of 233 patients with a minimum follow up of 10 years. Haematologica. 2013;98:875-80.

16 Bussel JB, Kaufmann CP, Ware RE, Woloski BM. Do the acute platelet responses of pa- tients with immune thrombocytopenic purpura (ITP) to IV anti-D and to IV gammaglobulin predict response to subsequent splenectomy? Am J Hematol. 2001;67:2733.

17 Choi CW, Kim BS, Seo JH, Shin SW, Kim YH, Kim JS, et al. Response to high-dose intravenous immune globulin as a valuable factor predicting the effect of splenectomy in chronic idiopathic thrombocytopenic purpura patients. Am J Hematol. 2001;66:197-202.

18 Shiino Y, Takahashi N, Okamoto T, Ishii Y, Yanagisawa A, Inagaki Y, et al. Surgical treatments of chronic idiopathic thrombocytopenic purpura and prognostic factors for splenectomy. Int Surg. 1996;81:140-3.

19 Ruivard M, Caulier MT, Vantelon JM, Tournilhac O, Schaeffer A, Godeau B, et al. The response to high-dose intravenous immunoglobulin or steroids is not predictive of outcome after splenectomy in adults with autoimmune thrombocytopenic purpura. $\mathrm{Br} \mathrm{J}$ Haematol. 1999;105:1130-2.

20 Radaelli F, Faccini P, Goldaniga M, Guggiari E, Pozzoli E, Maiolo AT, et al. Factors predicting response to splenectomy in adult patients with idiopathic thrombocytopenic purpura. Haematologica. 2000;85:1040-4.

21 Kwon HC, Moon CH, Cho YR, Kim MC, Kim $\mathrm{KH}$, Han JY, et al. Prognostic factors of response to laparoscopic splenectomy in pa- tients with idiopathic thrombocytopenic purpura. J Korean Med Sci. 2005;20:417-20.

22 Kuwana M, Okazaki Y, Ikeda Y. Splenic macrophages maintain the anti-platelet autoimmune response via uptake of opsonized platelets in patients with immune thrombocytopenic purpura. J Thromb Haemost. 2009;7:322-9.

23 Khodadi E, Asnafi AA, Shahrabi S, Shahjahani M, Saki N. Bone marrow niche in immune thrombocytopenia: a focus on megakaryopoiesis. Ann Hematol. 2016;95:1765-76.

24 Olsson B, Andersson PO, Jernås M, Jacobsson S, Carlsson B, Carlsson LM, et al. T-cell-mediated cytotoxicity toward platelets in chronic idiopathic thrombocytopenic purpura. Nat Med. 2003;9:1123-4.

25 Peng J, Ma SH, Liu J, Hou Y, Liu XM, Niu T, et al. Association of autoantibody specificity and response to intravenous immunoglobulin $\mathrm{G}$ therapy in immune thrombocytopenia: a multicenter cohort study. J Thromb Haemost. 2014;12:497-504.

26 Han JJ, Baek SK, Lee JJ, Kim SY, Cho KS, Yoon HJ. Long-term outcomes of a 5-year follow up of patients with immune thrombocytopenic purpura after splenectomy. Korean J Hematol. 2010;45:197-204.

27 Estcourt LJ, Birchall J, Allard S, Bassey SJ, Hersey P, Kerr JP, et al. Guidelines for the use of platelet transfusions. Br J Haematol. 2017; 176:365-94. 